\title{
Scabies Outbreak in an Intensive Care Unit with 1,659 Exposed Individuals-Key Factors for Controlling the Outbreak
}

\author{
Manuela Buehlmann, MD; Helmut Beltraminelli, MD; Christoph Strub, MD; Andreas Bircher, MD; Xavier Jordan, MD; \\ Manuel Battegay, MD; Peter Itin, MD; Andreas F. Widmer, MD, MS
}

овJестіVE. To investigate a large outbreak of scabies in an intensive care unit of a university hospital and an affiliated rehabilitation center, and to establish effective control measures to prevent further transmission.

DESIGN. Outbreak investigation.

SETTING. The intensive care unit of a 750-bed university hospital and an affiliated 92-bed rehabilitation center.

METHODS. All exposed individuals were screened by a senior staff dermatologist. Scabies was diagnosed on the basis of (1) identification of mites by skin scraping, (2) identification of mites by dermoscopy, or (3) clinical examination of patients without history of prior treatment for typical burrows. During a follow-up period of 6 months, the attack rate was calculated as the number of symptomatic individuals divided by the total number of exposed individuals.

INTERVENTIONs. All exposed healthcare workers (HCWs) and their household members underwent preemptive treatment. Initially, the most effective registered drug in Switzerland (ie, topical lindane) was prescribed, but this prescribption was switched to topical permethrin or systemic ivermectin as a result of the progression of the outbreak. Individuals with any signs or symptoms of scabies underwent dermatological examination.

RESUlTs. Within 7 months, 19 cases of scabies were diagnosed, 6 in children with a mean age of 3.1 years after exposure to the index patient with HIV and crusted scabies. A total of 1,640 exposed individuals underwent preemptive treatment. The highest attack rate of $26 \%-32 \%$ was observed among HCWs involved in the care of the index patient. A too-restricted definition of individuals at risk, noncompliance with treatment, and the limited effectiveness of lindane likely led to treatment failure, relapse, and reinfestation within families.

CONCLUSions. Crusted scabies resulted in high attack rates among HCWs and household contacts. Timely institution of hygienic precautions with close monitoring and widespread, simultaneous scabicide treatment of all exposed individuals are essential for control of an outbreak.

Infect Control Hosp Epidemiol 2009; 30:354-360

Scabies is a parasitic infection of the skin that is caused by the mite Sarcoptes scabiei var. hominis. Female mites burrow into the skin and lay up to 3 eggs per day in the epidermis. Clinical symptoms like skin rash and itching result from a delayed (2-6 weeks) host immune response to the mite. ${ }^{1}$ During the later stages in the course of the disease, scabies is diagnosed on the basis of clinical examination, dermoscopy, or skin scraping, but there is no reliable diagnostic test available for identifying the early stages of scabies. Person-toperson transmission occurs mainly by direct skin contact. ${ }^{2}$

Scabies occurs worldwide and is endemic in tropical and subtropical areas, where it is a major cause of secondary bacterial infection. Large epidemics of crusted scabies (also known as Norwegian scabies) appear to coincide with major wars and are related primarily to poverty and overcrowded living conditions. ${ }^{3}$ In industrialized countries, scabies occurs sporadically either in individuals or in outbreaks in healthcare institutions, such as long-term care facilities ${ }^{4,5}$ or acute care hospitals..$^{6-13}$ For the majority of outbreaks, the index patient is an individual with crusted scabies, a severe and highly contagious form of scabies typically affecting elderly, debilitated, neurologically impaired, or immunocompromised patients. Although caused by the same mite, crusted scabies differs from ordinary scabies in the number of mites present on the infected person $(1,000-1,000,000$ mites vs $10-15$ mites). ${ }^{1,3}$

There are several American and European guidelines for the treatment of individual cases of scabies. ${ }^{14,15}$ However, detailed

From the Department of Infectious Diseases and Hospital Epidemiology (M. Buehlmann, M. Battegay, A.F.W.) and the Department of Dermatology (H.B., C.T., A.B., P.I.), University Hospital Basel, and Rehab Basel (X.J.), Basel, Switzerland.

Received August 7, 2008; accepted November 7, 2008; electronically published March 2, 2009.

(C) 2009 by The Society for Healthcare Epidemiology of America. All rights reserved. 0899-823X/2009/3004-0007\$15.00. DOI: 10.1086/596113 
guidelines for the management of an epidemic of scabies in a hospital setting are scarce. ${ }^{14}$ In a large outbreak affecting 977 individuals, Obasanjo et al. ${ }^{10}$ found that the transmission of scabies among patients and healthcare workers (HCWs) was extensive, and they reported that the isolation recommendations from the Centers for Disease Control and Prevention were inadequate for patients who exhibited a more advanced stage of the disease.

We report a large outbreak of scabies involving 1,659 individuals and 2 acute care facilities with interdisciplinary activities in Switzerland, where scabies is not endemic. The outbreak lasted for several months and was controlled only after the implementation of strict infection control measures that went beyond the Centers for Disease Control and Prevention guidelines; these measures included prescription of multiple courses of drug treatment.

\section{METHODS}

\section{Setting}

In June 2007, several HCWs were exposed to a patient with unrecognized crusted scabies (ie, the index patient) who was treated in the intensive care unit (ICU) of the university hospital and then transferred to an affiliated rehabilitation center. The HCWs developed pruritus and rash after several weeks of contact with the index patient, triggering an outbreak investigation by the Department of Infectious Diseases and Hospital Epidemiology. The University Hospital Basel is a primary and tertiary care hospital with 750 beds, 3 ICUs, and approximately 27,000 admissions per year. The medical ICU that was involved in this outbreak has 19 beds. The rehabilitation center is an affiliated hospital with 92 beds and 1 ICU for patients with complex disabilities and/or spinal cord and brain injuries.

\section{Epidemiological Investigation}

Case identification. All HCWs who were exposed to the index patient and all patients who had either been exposed on the same ward or were cared for by the same HCWs as the index patient were identified by reviews of the lists of employees and patients and of the medical charts of both the ICU and the rehabilitation center.

Case definition. Confirmed scabies was defined as scabies diagnosed (exclusively by 3 senior staff members of the dermatology unit) on the basis of the identification of mites by skin scraping or dermoscopy or by clinical examination of patients without a prior history of scabies treatment for the presence of symptoms (eg, burrow-like lesions).

Questionnaire and attack rate. A standardized, electronically readable questionnaire was administered to all exposed HCWs while the outbreak was ongoing. This questionnaire was distributed to each HCW per treatment episode (ie, in the summer and autumn months). The attack rate for HCWs was calculated by dividing the number of symptomatic HCWs (ie, those with pruritus and/or skin rash) by the number of all exposed HCWs. Direct contact with the index patient was defined as involvement in diagnostic procedures or care of the index patient.

Treatment and preemptive treatment of scabies. Initially, topical lindane, the most effective registered drug for treatment of scabies in Switzerland, was selected as the primary drug for treatment on days 1-3 and days 8-10 of the outbreak. Lindane treatment failed to stop the progression of the outbreak; therefore, first-line treatment was switched to topical permethrin $5 \%$ on days 1 and 8 and oral ivermectin $(200 \mu \mathrm{g} /$ $\mathrm{kg}$ ) on day 1 and 14, because both are considered the most potent, currently available drugs for the treatment of scabies, although they are not registered in Switzerland for the treatment of scabies (off-label use).

\section{Data Analysis}

Data from the case-report form were scanned, electronically read, transferred to a spreadsheet program (Excel; Microsoft), and imported into SPSS, version 14.0.1 (SPSS), for statistical analysis. Categorical data were compared by use of the $\chi^{2}$ test.

\section{RESULTS}

\section{Epidemiological Investigation}

In April 2007, a patient with AIDS was admitted to the medical ICU at the university hospital for severe sepsis and pneumonia. Shortly after admission, a pruritic skin rash developed. A skin scraping, which was performed by the consultant for dermatology, failed to identify mites at this time, and rash was interpreted as a delayed hypersensitivity reaction to antibiotic treatment. After a 7-week stay in the ICU with standard precautions, the patient was transferred to an affiliated rehabilitation center on June 7,2007 . On June 13, a dermatologist was consulted for persistent pruritic skin rash, and crusted scabies was diagnosed. Contact isolation was initiated immediately, and the patient was treated with topical lindane and oral ivermectin on days 1,8 , and 15 . Scabies was subsequently diagnosed in 19 individuals, 12 at the university hospital and 7 at the rehabilitation center.

After diagnosis of crusted scabies in the index patient, several HCWs who worked in the ICU reported that they had developed pruritus. Scabies was diagnosed in 2 of the HCWs (a nurse and a physiotherapist) and in 2 household members of HCWs (a child and a spouse) (Figure). These HCWs whose household members received a diagnosis of scabies did not show any signs of scabies during their dermatological examination. In mid-August, 2007 (ie, 8 weeks after the first case of scabies was reported), scabies was diagnosed in 2 more nurses and a child. One nurse and the child of a HCW did not follow the recommendation of hospital epidemiology to undergo preemptive treatment with a scabicide in June 2007.

In November 2007, after 2 months without any new cases 

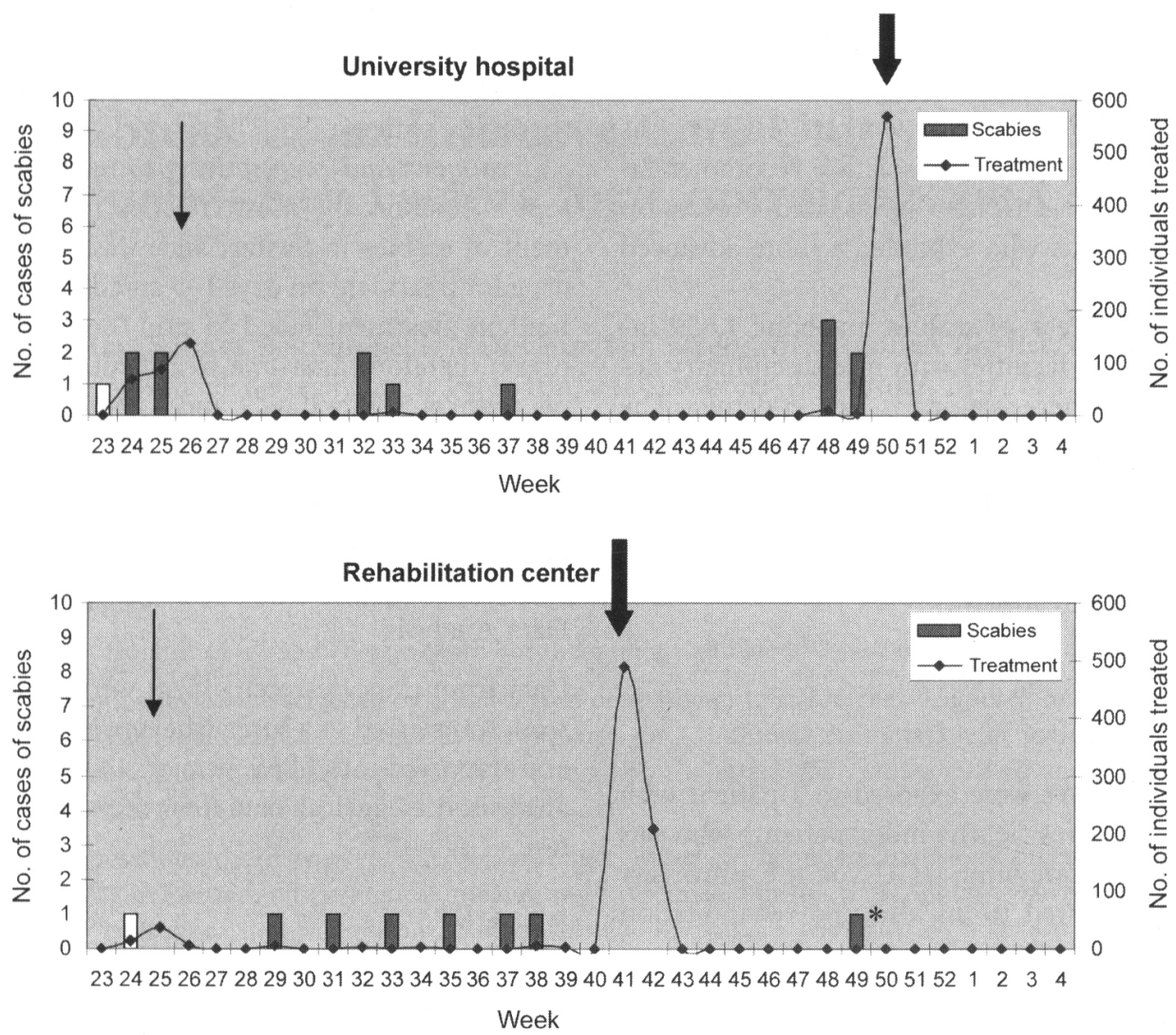

FIGURE. Number of confirmed cases of scabies and number of individuals treated with a scabicide (preemptively or for confirmed scabies) during a large outbreak of scabies in 2007 involving 1,659 individuals and 2 acute care facilities with interdisciplinary activities in Switzerland. The arrows indicate the implementation of an infection control intervention (ie, preemptive treatment or infection control measures); the size of the arrow represents the magnitude of the intervention. The asterisk indicates that scabies was detected in a household member living abroad (eg, in the grandfather of a child whose scabies was diagnosed in August).

of scabies, a 5-year-old son of a nurse who was exposed to the index patient during the outbreak presented with scabies. $\mathrm{He}$ and his parents had been preemptively treated with lindane in June. However, the parents did not treat their son's 5 other siblings, as recommended by hospital epidemiologists. During a dermatological examination of the household's contacts in November, scabies was also diagnosed in 1 sister and the mother.

At the end of November, scabies was diagnosed in 2 other nurses at the university hospital. One of them did not undergo preemptive treatment in June. Another nurse presented with a relapse, despite completed treatment with permethrin.

At the rehabilitation center, at the end of July 2007, 1 month after scabies was diagnosed in the index patient, scabies was diagnosed in a radiologist. Two weeks later, a second case of scabies was observed in a physiotherapist. Both of these HCWs had not applied scabicide treatment after exposure to the index patient, as recommended by hospital epidemiology in June. None of the 52 patients who had been in contact with the radiologist or physiotherapist during the incubation period developed scabies.
At the end of August, a 6-year-old son of a nurse presented with scabies. This nurse (ie, the son's mother) underwent a dermatological examination, which included dermoscopy, that resulted in a negative test result for scabies. The child had not received preemptive treatment with a scabicide in June after his mother's contact with the index patient, because he stayed with his grandparents at the time. More than 2 months later, scabies was diagnosed in the child's grandfather.

In September, 3 months after scabies was diagnosed in the index patient, scabies was diagnosed in 2 patients. One of them had documented exposure to the above-mentioned radiologist and nurse. In late September, scabies was diagnosed in the child of an occupational therapy practitioner, despite having undergone preemptive treatment in June. The occupational therapy practitioner's whole family had been preemptively treated with lindane in June after the occupational therapy practitioner's contact with the index patient. Preemptive treatment of all children staying in the same kindergarten as the child and with the same nanny was initiated by local health authorities.

In summary, within 7 months, 1,640 individuals were ex- 
posed and preemptively treated with a scabicide, 875 in the university hospital and 765 in the rehabilitation center. Nineteen cases of confirmed scabies were diagnosed in both healthcare facilities, 2 individuals had a relapse and had to be treated twice (Table 1).

Of the 9 household members with a diagnosis of scabies, $5(56 \%)$ were small children with a mean age of 3.2 years. Only 1 child had been treated with lindane at the beginning of the epidemic in June, because he presented with a case of scabies that was at a later stage in the course of the disease. Four of the other household members had not been treated preemptively, as recommended, and 1 had received inadequate treatment (with crotamiton) at that time. There were 2 cases of probable failure of preemptive treatment with lindane (one involving an $\mathrm{HCW}$ and the other a 4-year-old child).

\section{Infection Control Measures}

The index patient was considered to be infested and highly infectious since May 1, 2007, on the basis of the finding that the skin scrapings of the index patient tested negative for scabies at the beginning of April, and highly infectious crusted scabies takes at least 2-3 mite generations to develop (ie, 34 weeks). ${ }^{1}$ The following targeted infection control measures were taken as soon as the outbreak was identified.

Regardless of symptoms, all exposed HCWs and their household contacts were preemptively treated with a scabicide. Asymptomatic household contacts were not treated initially at the rehabilitation center. A dermatological examination was performed for persons with pruritus and/or skin rash, women who were pregnant, persons with chronic skin disease, persons with immunosuppression, and children less than 3 years old. All HCWs wore protective gowns and gloves (contact precautions) while working, until they had completed their preemptive scabicide treatment.

For the index patient, contact isolation was maintained until completion of the second treatment course: Examination by a dermatologist was performed for patients who were hospitalized on the same ward and, therefore, cared for by the same HCWs as the index patient and had been in contact with infected HCWs. Initially, no preemptive treatment was administered to asymptomatic patients.

After the outbreak had continued for more than 3 months, a stricter approach was initiated in both healthcare facilities: all HCWs of the ICU and their household contacts, all staff members working temporarily on the wards involved in the outbreak, such as radiologists and therapists, as well as all patients and their families were treated preemptively with a scabicide on the same day. Thereafter, no further cases of scabies were diagnosed during a follow-up period of 6 months. Discharged patients who had been treated in these 2 healthcare facilities during the previous 8 weeks were in-

TABLE 1. Data on Confirmed Cases of Scabies at 2 Acute Care Facilities in Switzerland in 2007

\begin{tabular}{|c|c|c|c|c|c|c|}
\hline $\begin{array}{l}\text { Healthcare facility, } \\
\text { individual infected }\end{array}$ & $\begin{array}{l}\text { Age, } \\
\text { years }\end{array}$ & Sex & $\begin{array}{c}\text { Date of } \\
\text { diagnosis }\end{array}$ & $\begin{array}{c}\text { No. of days } \\
\text { after index } \\
\text { patient identified }\end{array}$ & $\begin{array}{l}\text { Preemptive } \\
\text { treatment }\end{array}$ & $\begin{array}{l}\text { Treatment for } \\
\text { confirmed scabies }\end{array}$ \\
\hline \multicolumn{7}{|l|}{ University hospital } \\
\hline Household member & 46 & $\mathrm{~F}$ & June 16 & 3 & None & Lindane \\
\hline Household member & 2 & $\mathrm{M}$ & June 17 & 4 & None & Lindane \\
\hline HCW nurse & 31 & $\mathbf{F}$ & June 19 & 6 & None & Lindane, permethrin \\
\hline Physiotherapist & 44 & $\mathrm{M}$ & June 22 & 9 & None & Lindane \\
\hline Household member & 0.7 & $\mathbf{F}$ & August 10 & 58 & None & Permethrin \\
\hline HCW nurse ${ }^{a}$ & 30 & $\mathrm{M}$ & August 10 & 58 & None & Permethrin \\
\hline HCW nurse & 53 & $\mathbf{F}$ & August 13 & 61 & Lindane & Permethrin \\
\hline Household member & 2 & F & November 28 & 168 & None & Permethrin \\
\hline Household member & 5 & $\mathrm{M}$ & November 28 & 168 & Crotamiton & Permethrin \\
\hline Household member & 37 & $\mathrm{~F}$ & November 28 & 168 & Crotamiton & Permethrin, ivermectin \\
\hline HCW nurse & 50 & M & December 6 & 176 & None & Permethrin, ivermectin \\
\hline HCW nurse & 44 & $\mathrm{M}$ & December 6 & 176 & None & Permethrin \\
\hline \multicolumn{7}{|l|}{ Rehabilitation center } \\
\hline $\begin{array}{l}\text { Staff member of radiology } \\
\text { department }\end{array}$ & 56 & $\mathrm{M}$ & July 26 & 43 & None & Lindane \\
\hline HCW physiotherapist & 56 & $\mathrm{M}$ & August 6 & 54 & None & Lindane \\
\hline Household member ${ }^{\mathrm{b}}$ & 6 & $\mathrm{M}$ & August 22 & 70 & None & Permethrin \\
\hline Patient & 53 & $\mathrm{M}$ & September 4 & 98 & None & Ivermectin \\
\hline Patient & 25 & $\mathrm{M}$ & September 19 & 83 & None & Ivermectin \\
\hline Household member & 4 & M & September 24 & 103 & Lindane & Permethrin \\
\hline Household member & Unknown & M & December 12 & Unknown & None & Ivermectin \\
\hline
\end{tabular}

NOTE. All preemptive treatments failed. HCW, healthcare worker; household member, household member of HCW.

${ }^{a}$ Relapse of scabies on December 5.

b Relapse of scabies on September 17. 
formed to contact their dermatologist if they experienced signs or symptoms of scabies, such as pruritus or exanthema.

\section{Questionnaire}

Of 391 questionnaires that were distributed to the HCWs at the university hospital, 307 (79\%) were returned. Of 307 HCWs who participated, 92 (30\%) were male, 134 (44\%) were nurses, $85(28 \%)$ were staff members of the radiology department, $46(15 \%)$ were physicians, and $22(7 \%)$ were therapists. In June 2007 , during the first treatment episode, $68(59 \%)$ of 115 HCWs reported having been in direct contact with the index patient. The attack rates are shown in Table 2. Of the 307 HCWs who responded to the questionnaire, $287(93 \%)$ reported having been treated for scabies (24 [82\%] of the 287 HCWs in June and all 287 HCWs [100\%] in December): 90 (29\%) were treated with lindane, $91(30 \%)$ were treated with permethrin (at a concentration of 5\%), 98 $(32 \%)$ were treated with ivermectin, and $4(1 \%)$ were treated with permethrin and ivermectin. There were no serious adverse side effects observed.

Of the 278 questionnaires that were distributed to the HCWs at the rehabilitation center, $251(90 \%)$ were returned. Of $251 \mathrm{HCWs}$ who participated, $60(24 \%)$ were male, 150 $(60 \%)$ were nurses, $46(18 \%)$ were therapists, and $22(9 \%)$ were physicians. Although $32(91 \%)$ of the $35 \mathrm{HCWs}$ who participated had been in direct contact with the index patient in June, only $57(26 \%)$ of 216 HCWs reported having been in direct contact with the index patient in September. The attack rate is shown in Table 2. All HCWs reported having performed treatment as recommended; of the 251 HCWs who participated, $54(22 \%)$ were treated with lindane, 195 (78\%) were treated with permethrin, and $27(11 \%)$ were treated with ivermectin.

\section{Costs}

The total financial cost of the outbreak could not be determined. However, the direct cost for medication and dermatological consultation was $\$ 47,280$ US dollars. The staff at the university hospital worked more than 1,200 hours managing the scabies epidemic.

\section{DISCUSSION}

To our knowledge, this was one of the largest hospital outbreaks of scabies reported in the literature, with 1,659 exposed individuals from 1 point source (an ICU patient). The highest attack rates (ie, 26\%-32\%) were observed for HCWs with direct contact with the index patient, with $5 \%-9 \%$ of the household members of these HCWs being infected as well. These attack rates are similar to those reported in the literature (ie, $34 \%$ of HCWs and $28 \%$ of household members). ${ }^{7,16}$ In contrast, during outbreaks of scabies, the attack rates reported in the literature were usually high (mean rate, $13 \%)^{16}$ for patients in acute care settings, compared with the attack rate for the exposed patients reported in our study (ie, less than $2 \%$ ). In fact, only 2 patients were identified with scabies during the whole outbreak. The risk of transmission may have been lower in our healthcare settings, because the patients in the ICU and the patients with severe brain injury at the rehabilitation center almost never have direct contact with each other.

Our study demonstrates that the timely initiation of wide-

TABLE 2. Attack Rates of Scabies at 2 Acute Care Facilities in Switzerland in 2007, Stratified for Healthcare Facility and Type of Contact

\begin{tabular}{lc}
\hline Healthcare facility, individuals infected & $\begin{array}{c}\text { Proportion (\%) of } \\
\text { symptomatic individuals }\end{array}$ \\
\hline University hospital & \\
HCWs & $32 / 307(10)$ \\
With direct contact with index patient ${ }^{\mathrm{a}}$ & $17 / 64(27)$ \\
With no direct contact with index patient ${ }^{\mathrm{b}}$ & $3 / 41(7)$ \\
Household members of HCWs & $28 / 307(9)$ \\
Patients & $0 / 79(0)$ \\
Rehabilitation center & \\
HCWs & \\
With direct contact with index patient & $21 / 65(32)$ \\
With no contact with index patient & $0 / 109(0)$ \\
Household members of HCWs & $18 / 344(5)$ \\
Patients & $2 / 74(3)$ \\
\hline
\end{tabular}

NOTE. The attack rate was calculated as the number of symptomatic individuals divided by the total number of exposed individuals. Symptomatic individuals are defined as those with pruritus and/or exanthema. $\mathrm{HCW}$, healthcare worker.

${ }^{a}$ Because the index patient was hospitalized in June, the analysis that stratified for individuals with and without direct contact with the index patient could only be performed for the first treatment episode in June 2007.

b The number of contact patients was recorded only for the second treatment episode in October 2007. 
spread infection control measures, such as isolation precautions and preemptive treatment of contact persons, is essential for outbreak control. As a result of the high infectivity of crusted scabies, symptoms developed in $7 \%$ of the HCWs who probably had very limited contact with the index patient, and a later stage of scabies was diagnosed in one such HCW. Focusing on the preemptive treatment of high-risk HCWs, as suggested by Obasanjo et al. ${ }^{10}$ may therefore not be sufficient.

In retrospect, the number of preemptively treated individuals at the rehabilitation center in June 2007 was too small, resulting in several additional cases of scabies in HCWs, household members, and patients. At the university hospital, treatment was not performed simultaneously for all individuals, leaving the opportunity for reinfestation in the hospital and-probably more important-within families. Also, despite the extensive campaign to inform HCWs by mail, flyers, and meetings about infection control measures, including preemptive scabicide treatment, $16 \%$ of HCWs did not participate, resulting in scabies transmission within families. In addition, treatment with lindane-the only registered drug for treatment of scabies in Switzerland, besides crotamiton, but not the most effective-may have contributed to the failure to control the first wave of the outbreak.

The key to the successful control of the outbreak was the simultaneous (ie, more than $80 \%$ of individual were treated on the same day), strict, and widespread treatment of all exposed individuals with the most potent agents currently available (namely, topical permethrin $5 \%$ and oral ivermectin), ${ }^{17,18}$ even if these agents were not registered in our country for this indication. The rate of compliance and the effectiveness of treatment increased, particularly with the use of oral ivermectin, as was also reported elsewhere..$^{10} \mathrm{An}$ additional component was limiting the number of dermatologists who screened patients and HCWs for the presence of scabies. Even boardcertified dermatologists misdiagnosed cases, and it became clear that early diagnosis of scabies is very difficult, if not impossible.

Interestingly, 5 (56\%) of the 9 household members with a confirmed diagnosis of scabies were small children with a mean age of 3.2 years. In previous epidemiological studies, the highest incidence of scabies in the community was found in children and teenagers 6-24 years of age. ${ }^{19,20}$ Children are highly susceptible to the acquisition of scabies because of their close exposure to parents and siblings and their thinner skin and stratum corneum. Also, because parents have concerns about the potential side effects of preemptively treating their children with these potent agent, treatment is delayed, thus increasing their children's risk of acquiring scabies. Initially, lindane, the most effective scabicide approved in Switzerland, was used for preemptive treatment. However, it is contraindicated for children less than 3 years of age, because of potential neurotoxicity ${ }^{21}$; this may have delayed treatment and decreased compliance. To our knowledge, our study is the first to show that the infestation of small children by S. scabiei is a key factor in healthcare-related outbreaks with scabies, thus explaining reinfestation within families.

Our study had several limitations. First, the exact time of the start of symptoms for all involved individuals could not be determined. Second, attack rates were estimated on the basis of the number of symptomatic individuals rather than determined on the basis of the number of proven cases of scabies, which could have potentially led to an overestimation of the extent of the outbreak. However, we may also have underestimated the magnitude of the outbreak, because there was no test for early diagnosis of scabies. ${ }^{3}$ In a former study, $64 \%$ of HCWs were reported to have symptoms or signs of scabies, such as pruritus or papules, and only $23 \%$ of HCWs had skin scrapings that indicated the presence of S. scabiei. ${ }^{8}$ Despite these limitations, the attack rates in our study are comparable to those of former outbreak studies, in which similar case definitions, such as the presence of a pruritic skin rash, were used. ${ }^{7,10}$ Third, epidemiological data were based on questionnaires with the potential for bias, but the high response rates (ie, $78 \%-90 \%$ of HCWs responded) are considered to be representantive.

In conclusion, the key factors for controlling the outbreak of scabies in an acute care setting were (1) the widespread preemptive treatment of all potentially exposed individuals; (2) offering the most effective treatment, either with topical permethrin or oral ivermectin; (3) initiating of treatment on the same day for most, if not all, individuals who qualified for treatment; and (4) paying special attention to children. Finally, close monitoring of infection control measures should continue as long as the outbreak is not under control.

\section{ACKNOWLEDGMENTS}

Our study is dedicated to Theo Rufli, Professor of Dermatology emeritus at the University Hospital Basel.

We thank Rainer Herold and Marc Dangel of the Department of Infectious Diseases and Hospital Epidemiology at the University Hospital of Basel, Rosaria De Lorenzo (study nurse of the Department of Dermatology), Angelika Lehmann (head nurse of the ICU), and Doris Mahler (infection control nurse in the rehabilitation facility) for their contribution in data collection and their efforts to control the outbreak.

Potential conflicts of interest. All authors report no conflicts of interest relevant to this article.

Address reprint requests to Andreas F. Widmer, MD, MS, Department of Infectious Diseases and Hospital Epidemiology, University Hospital Basel, Petersgraben 4, CH-4031 Basel, Switzerland (awidmer@uhbs.ch).

Presented in part: 18th Annual Congress of the European Society of Clinical Microbiology and Infectious Diseases; Barcelona, Spain; April 19-22, 2008.

\section{REFERENCES}

1. Hengge UR, Currie BJ, Jager G, Lupi O, Schwartz RA. Scabies: a ubiquitous neglected skin disease. Lancet Infect Dis 2006; 6:769-779.

2. Heukelbach J, Feldmeier H. Scabies. Lancet 2006; 367:1767-1774. 
3. Walton SF, Currie BJ. Problems in diagnosing scabies, a global disease in human and animal populations. Clin Microbiol Rev 2007; 20:268-279.

4. Andersen BM, Haugen H, Rasch M, Heldal HA, Tageson A. Outbreak of scabies in Norwegian nursing homes and home care patients: control and prevention. J Hosp Infect 2000; 45:160-164.

5. Degelau J. Scabies in long-term care facilities. Infect Control Hosp Epidemiol 1992; 13:421-425.

6. Clark J, Friesen DL, Williams WA. Management of an outbreak of Norwegian scabies. Am I Infect Control 1992; 20:217-220.

7. Fajardo-Velázquez R, Urdez-Hernández E, Ysita-Morales A. Nosocomial outbreak of scabies from a Norwegian scabies case [in Spanish]. Salud Publica Mex 2004; 46:251-254.

8. Garcia C, Iglesias D, Terashima A, Canales M, Gotuzzo E. Use of ivermectin to treat an institutional outbreak of scabies in a low-resource setting. Infect Control Hosp Epidemiol 2007; 28:1337-1338.

9. Larrosa A, Cortes-Blanco M, Martinez S, et al. Nosocomial outbreak of scabies in a hospital in Spain. Euro Surveill 2003; 8:199-203.

10. Obasanjo $\mathrm{OO}, \mathrm{Wu} \mathrm{P}$, Conlon $\mathrm{M}$, et al. An outbreak of scabies in a teaching hospital: lessons learned. Infect Control Hosp Epidemiol 2001; 22:13-18.

11. Pasternak J, Richtmann R, Ganme AP, et al. Scabies epidemic: price and prejudice. Infect Control Hosp Epidemiol 1994; 15:540-542.

12. Scalzini A, Barni C, Bertelli D, Sueri L. A hospital outbreak of scabies. J Hosp Infect 1992; 22:167-168.
13. Sirera G, Rius F, Romeu J, et al. Hospital outbreak of scabies stemming from two AIDS patients with Norwegian scabies. Lancet 1990; 335:1227.

14. Sunderkotter C, Mayser P, Folster-Holst R, Maier WA, Kampen H, Hamm H. Scabies. J Dtsch Dermatol Ges 2007; 5:424-430.

15. Workowski KA, Berman SM; Centers for Disease Control and Prevention (CDC). Sexually transmitted diseases treatment guidelines, 2006 (published correction appears in MMWR Recomm Rep 2006; 55:997). MMWR Recomm Rep 2006; 55(RR-11):1-94.

16. Vorou R, Remoudaki HD, Maltezou HC. Nosocomial scabies. J Hosp Infect 2007; 65:9-14.

17. Strong M, Johnstone PW. Interventions for treating scabies. Cochrane Database Syst Rev 2007; (3):CD000320.

18. Tjioe $M$, Vissers WH. Scabies outbreaks in nursing homes for the elderly: recognition, treatment options and control of reinfestation. Drugs Aging 2008; 25:299-306.

19. Buczek A, Pabis B, Bartosik K, Stanislawek IM, Salata M, Pabis A. Epidemiological study of scabies in different environmental conditions in central Poland. Ann Epidemiol 2006; 16:423-428.

20. Pannell RS, Fleming DM, Cross KW. The incidence of molluscum contagiosum, scabies and lichen planus. Epidemiol Infect 2005; 133:985-991.

21. Singal A, Thami GP. Lindane neurotoxicity in childhood. Am J Ther 2006; 13:277-280. 